\title{
INTEGRIDADE DA PELE EM IDOSOS: REVISÃO DA LITERATURA SEGUNDO AS CARTAS DE PROMOÇÃO DA SAÚDE
}

Ana Luisa Brandão de Carvalho Lira ${ }^{1}$, Jéssica Dantas de Sá2, Isadora Lorenna Alves Nogueira ${ }^{3}$, Marina Dantas

Cardoso de Medeiros ${ }^{4}$, Maria Isabel da Conceição Dias Fernandes ${ }^{5}$, Allyne Fortes Vitor ${ }^{6}$

RESUMO: Trata-se de uma revisão bibliográfica que objetivou relacionar as medidas educativas para a promoção da integridade da pele em idosos com as Cartas de Promoção da Saúde. Realizou-se a busca nas bases de dados CINAHL, SCOPUS, LILACS e COCHRANE, nos portais CAPES e BVS e na biblioteca PUBMED, mediante a aplicação dos descritores Health Education; Skin e Aged. Os resultados dos 7 artigos analisados apontaram como principais medidas educativas: inspeção diária da pele, cuidados com calçados e com os pés, uso regular de protetor solar e mudanças de decúbito. Essas medidas estavam relacionadas com as seguintes Cartas de Promoção da Saúde: Ottawa, Declaração de Santafé de Bogotá e Declaração de Jacarta. Conclui-se que as medidas educativas, baseadas nas Cartas, são de grande relevância para a criação de uma cultura de saúde, com enfoque na população e no indivíduo como agentes executores imprescindíveis para o alcance da promoção da saúde.

PALAVRAS-CHAVE: Enfermagem; Promoção da saúde; Educação em saúde; Pele; Idoso.

\section{SKIN INTEGRITY IN OLDER ADULTS: LITERATURE REVIEW ACCORDING TO HEALTH PROMOTION CHARTERS}

ABSTRACT: This bibliographical review aimed to relate educational measures for the promotion of skin integrity in older adults with the Health Promotion Charters. The search was performed in the databases CINAHL, SCOPUS, LILACS and COCHRANE, in the CAPES and BVS portals and in the PUBMED library, using the descriptors Health Education; Skin and Aged. The results from the 7 articles analyzed indicate, as the principle educative measures, daily inspection of the skin, care with footwear and the feet, regular use of sunblock and changes in supine position. These measures were related to the following Charters for Health Promotion: Ottawa, the Declaration of Bogotá and the Jakarta Declaration. It is concluded that educational measures, based on the Charters, are highly relevant for the creation of a culture of health, with focus on the population and individual as essential operative agents for the achievement of health promotion.

KEYWORDS: Nursing; Health promotion; Health education; Skin; Older adult.

\section{INTEGRIDAD DE LA PIEL EN ANCIANOS: REVISIÓN DE LITERATURA DE ACUERDO CON LAS CARTAS DE PROMOCIÓN DE LA SALUD}

RESUMEN: Esta es una revisión bibliográfica cuyo objetivo fue relacionar las medidas educativas para la promoción de la integridad de la piel en ancianos por medio de las Cartas de Promoción de la Salud. La búsqueda fue realizada en las bases de datos CINAHL, SCOPUS, LILACS y COCHRANE, en portales CAPES y BVS y en la biblioteca PUBMED, por medio de la aplicación de los descriptores Health Education; Skin y Aged. Los resultados de los 7 artículos analizados apuntan como principales medidas educativas: inspección no diaria de la piel, cuidados con zapatos y con los pies, uso regular de protector solar y cambios de decúbito. Tales medidas estaban relacionadas con las siguientes Cartas de Promoción de la Salud: Ottawa, Declaración de Santafé de Bogotá y Declaración de Jacarta. La conclusión es de que las medidas educativas, basadas en las Cartas, tienen gran relevancia para la creación de una cultura de salud, con enfoque en la población y en el individuo como agentes ejecutores imprescindibles para llegar a la promoción de la salud.

PALABRAS CLAVE: Enfermería; Promoción de la salud; Educación en salud; Piel; Anciano.

\footnotetext{
${ }^{1}$ Enfermeira. Doutora em Enfermagem. Professora do Departamento e do Programa de Pós-Graduação em Enfermagem da Universidade Federal do Rio Grande do Norte - UFRN.

${ }^{2}$ Acadêmica do Curso de Graduação em Enfermagem da UFRN. Bolsista CNPq de Graduação Sanduíche no exterior.

${ }^{3}$ Acadêmica do Curso de Graduação em Enfermagem da UFRN. Bolsista PET.

${ }^{4}$ Acadêmica do Curso de Graduação em Enfermagem da UFRN.

${ }^{5}$ Enfermeira. Mestranda pelo Programa de Pós-Graduação em Enfermagem da UFRN. Bolsista REUNI.

${ }^{6}$ Enfermeira. Doutora em Enfermagem. Professora do Departamento de Enfermagem da UFRN.
}

Autor correspondente:

Ana Luisa Brandão de Carvalho Lira

Universidade Federal do Rio Grande do Norte

BR 101, s/n - 59072-970 - Natal-RN-Brasil

E-mail: analira@ufrnet.br
Recebido: 10/07/2011 Aprovado: 06/06/2012 


\section{INTRODUÇÃO}

O desenvolvimento econômico, o avanço da industrialização e, principalmente, da Medicina, constituem nova realidade na qual o aumento da expectativa de vida populacional tem corroborado para uma população cada vez mais idosa. Diante do crescente número de idosos em todo o mundo, a necessidade de cuidados de saúde especializados para essa faixa etária emerge rapidamente ${ }^{(1)}$.

Assim, o processo de Enfermagem Gerontogeriátrica, com base na Política Nacional do Idoso, deverá fundamentar a observação da figura humana do idoso sob a ótica de uma contextualização que contemple sua diversidade e individualidade. Devem-se considerar os aspectos biopsicossociais e espirituais vivenciados pelo idoso e pela família, como clientes da enfermagem, promovendo um viver saudável, compensando limitações e incapacidades, proporcionando tratamento e cuidado específicos e facilitação do processo de cuidar ${ }^{(2)}$.

O envelhecimento não é conceituado como doença, mas pode determinar grande número de transtornos, sendo as mudanças produzidas no aspecto da pele um dos sinais mais evidentes. A pele é o maior e mais visível órgão do corpo humano, reflete condições físicas e psicológicas, tais como saúde, idade, diferenças étnicas e culturais. É responsável pela proteção, excreção, regulação da temperatura, sustentação, percepção sensitiva, metabolismo de vitamina $\mathrm{D}$, secreção, comunicação, hemostasia, absorção e identificação ${ }^{(3)}$.

Portanto, é fundamental a manutenção da pele íntegra na defesa do organismo frente às alterações diversas, traumatismos mecânicos, térmicos, químicos, radiação ou situações múltiplas a que o ser humano está exposto durante toda sua vida ${ }^{(4)}$. Dessa forma, a pele do idoso necessita de um cuidado especial, visto que o risco de lesão nesta faixa etária aumenta em virtude da diminuição da função de barreira, como também da maior dificuldade em perceber estímulos traumáticos e agressivos, devido a capacidade reduzida dos receptores sensoriais ${ }^{(4)}$.

Os principais problemas de pele em idosos são as dermatites, infecção por fungos, prurido, tumores benignos e infecções virais, bem como o câncer de pele em função de anos de exposição solar sem proteção ${ }^{(1)}$. Além desses, a úlcera de pé é percebida como uma das mais importantes complicações do diabetes e, sendo a tipo II predominante em idosos, o estudo do rompimento da integridade cutânea nesses pacientes passa a ser de fundamental importância ${ }^{(5)}$.

A úlcera por pressão constitui-se também como fator relevante para o rompimento da integridade da pele, evidenciado em pacientes com dificuldades de locomoção, realidade comum em idosos ${ }^{(6)}$.

Os problemas cutâneos supracitados normalmente possuem caráter evitável por meio de medidas preventivas. A educação em saúde desenvolvida pela enfermagem torna-se, portanto, ponto primordial uma vez que provocará maior interesse do paciente em aprender sobre os cuidados necessários para a manutenção da sua saúde, promovendo assim a integridade da pele ${ }^{(5)}$. Ademais, a promoção da saúde em idosos reduz a mortalidade antecipada, bem como propicia melhor qualidade de vida ${ }^{(6)}$, desse modo, é necessário enfoque da promoção da saúde na perspectiva da educação em saúde no âmbito da pele senil.

Nesse aspecto, as Cartas de Promoção da Saúde contribuem diretamente com este processo, na medida em que priorizam a capacitação da comunidade e do indivíduo acerca das medidas práticas primordiais à garantia de um estilo de vida saudável. Direcionam-se à educação em saúde com enfoque na população e indivíduo como agentes executores imprescindíveis de tais ações e, concomitantemente, promovem avanços significativos sobre a temática mundialmente ${ }^{(7-8)}$.

Dessa forma, com o intuito de contribuir para a melhoria dos cuidados em saúde e da qualidade de vida dos idosos, o presente trabalho tem como objetivo relacionar as medidas educativas para a promoção da integridade da pele em idosos com as Cartas de Promoção da Saúde.

\section{MÉTODO}

Trata-se de uma revisão bibliográfica que objetivou relacionar as medidas educativas para a promoção da integridade da pele em idosos com as Cartas de Promoção da Saúde. Nesse sentido, a pesquisa bibliográfica não é mera repetição do que já foi dito ou escrito sobre certo assunto, mas propicia o exame de um tema sob um enfoque de buscar, avaliar e sintetizar evidências disponíveis do tema investigado, aumentando a capacidade de generalização dos dados acerca de um fenômeno ${ }^{(9)}$.

Para tanto, foi utilizada a seguinte questão norteadora: Qual a relação entre as medidas educativas para a promoção da integridade da pele em pessoas idosas e as Cartas de Promoção da Saúde?

Para a seleção dos artigos, buscaram-se as bases de dados: SCOPUS, CINAHL (Cumulative Index to Nursing and Allied Health Literature), LILACS (Literatura

Cogitare Enferm. 2012 Out/Dez; 17(4):767-74 
Latino-Americana e do Caribe em Ciências da Saúde); os Portais da CAPES (Coordenação de Aperfeiçoamento de Pessoal de Nível Superior) e da BVS (Biblioteca Virtual em Saúde) e a biblioteca PUBMED (National Library of Medicine and Nattional Institutes of Health).

A busca ocorreu no período de novembro a dezembro de 2010, e durante este período cada base de dados e portal foi acessado em um único dia por três pesquisadores, simultaneamente e em computadores diferentes, a fim de garantir a seleção do maior número de artigos relevantes para a pesquisa. Foram utilizados os seguintes descritores: Health Education; Skin e Aged, mediante o cruzamento dos três descritores, conectados pelo operador booleano AND.

No tocante ao recorte temporal, foram captadas todas as publicações disponíveis em cada base de dados ou portal até o ano 2010, sem limite anterior, pela intenção de proporcionar amapla avaliação sobre o objeto do estudo.

Os critérios de inclusão estabelecidos foram: artigos completos disponíveis nas bases e portais selecionados; artigos disponíveis nos idiomas português, inglês ou espanhol; e artigos que abordam as medidas educativas para promoção da integridade da pele em pessoas idosas. Os critérios de exclusão foram: editoriais, cartas ao editor, artigos que não abordem a temática relevante ao alcance do objetivo da revisão; e artigos repetidos nas respectivas bases de dados e portais pesquisados.

Durante a pesquisa inicial, mediante a aplicação dos descritores, foram encontrados 9.583 artigos na CINAHL, 4.548 na SCOPUS, 554 na BVS, 326 no Portal da CAPES, 1.524 na PUBMED e nenhum na LILACS. Depois de concluída a etapa de coleta de dados inicial e aplicados os critérios de inclusão e exclusão mediante a leitura de cada artigo, a amostra constituiu-se de 7 artigos: dois eram da SCOPUS, um da CINAHL, um da BVS, dois do Portal CAPES e um da PUBMED.

Como estratégia para a análise e síntese dos dados, utilizou-se um instrumento composto por informações de identificação do estudo, instituição sede do estudo, tipo de revista científica, características metodológicas e avaliação do rigor metodológico do estudo.

A apresentação e a discussão dos achados foram realizadas de forma descritiva. Inicialmente houve a caracterização dos estudos e, posteriormente, a relação entre as medidas educativas e as recomendações das Cartas de Promoção da Saúde, a fim de obter um melhor entendimento dos resultados encontrados. Os estudos foram avaliados criticamente fundamentando-se nas Cartas de Promoção da Saúde ${ }^{(7)}$.

\section{RESULTADOS}

Inicialmente, é apresentado um panorama geral dos artigos avaliados. Quanto à nacionalidade, constatou-se que a maior parte $(28,57 \%)$ dos estudos foram realizados nos Estados Unidos, sendo o idioma predominante o inglês $(85,71 \%)$. Em relação ao ano, prevaleceram os artigos publicados nos anos de 2004 (28,57\%) e 2006 $(28,57 \%)$. Quanto à área, houve predomínio da Medicina $(42,86 \%)$ e da Enfermagem (42,86\%).

Quanto ao local de realização do estudo, a maioria dos artigos (71,43\%) não informou a instituição sede dos mesmos. O diagnóstico relacionado ao rompimento da integridade da pele com maior frequência foi úlcera neuropática diabética $(42,86 \%)$. Aoavaliaro nível de evidência dos artigos analisados, constatou-se que um artigo apresentou nível de evidência II, e os níveis III, IV e V foram contemplados por dois artigos cada.

O quadro 1 apresenta as recomendações presentes nas Cartas de Promoção da Saúde, no contexto de educação em saúde, bem como sua incidência de aparecimento nos artigos analisados. É importante esclarecer que algumas publicações apresentaram mais de uma recomendação da Carta de Ottawa. Conforme observado, destaca-se: Carta de Ottawa, com uma de suas recomendações presentes em todos os artigos e outra em apenas dois; a Declaração de Santafé de Bogotá, com sua recomendação em seis estudos e a Declaração de Jacarta, com sua recomendação em todos os artigos analisados. O Quadro 2 apresenta a síntese dos artigos analisados.

Quadro 1 - Recomendações das Cartas de Promoção da Saúde nos artigos analisados. Natal, 2010.

\begin{tabular}{|l|l|l|}
\hline $\begin{array}{l}\text { Carta de Pro- } \\
\text { moção da Saúde }\end{array}$ & \multicolumn{1}{|c|}{$\begin{array}{c}\text { Recomendações para } \\
\text { educação em saúde }\end{array}$} & N \\
\hline \multirow{2}{*}{$\begin{array}{l}\text { Carta de } \\
\text { Ottawa }\end{array}$} & $\begin{array}{l}\text { Reconhecer as pessoas como o } \\
\text { principal recurso para a saúde; } \\
\text { apoiá-las e capacitá-las para } \\
\text { que se mantenham saudáveis } \\
\text { a si próprias, às suas famílias } \\
\text { e amigos }\end{array}$ & 7 \\
\cline { 2 - 4 } $\begin{array}{l}\text { Reorientar os serviços de saúde } \\
\text { e os recursos disponíveis para a } \\
\text { promoção da saúde }\end{array}$ & 2 \\
\hline $\begin{array}{l}\text { Declaração de } \\
\text { Santafé de } \\
\text { Bogotá }\end{array}$ & $\begin{array}{l}\text { Impulsionar o conceito de saúde } \\
\text { e a promoção da saúde como } \\
\text { estratégia para modificar fatores } \\
\text { condicionantes }\end{array}$ & 6 \\
\hline $\begin{array}{l}\text { Declaração de } \\
\text { Jacarta }\end{array}$ & $\begin{array}{l}\text { Acumulação de conhecimentos } \\
\text { sobre as melhores práticas }\end{array}$ & 7 \\
\hline
\end{tabular}


Quadro 2 - Síntese dos artigos analisados segundo o tipo de estudo, a medida educativa utilizada e as recomendações das Cartas de Promoção da Saúde. Natal, 2010

\begin{tabular}{|c|c|c|}
\hline Tipo de estudo & Medidas educativas & $\begin{array}{l}\text { Recomendações das Cartas de } \\
\text { Promoção da Saúde }\end{array}$ \\
\hline $\begin{array}{l}\text { Estudo retrospec- } \\
\text { tivo transversal }{ }^{(1)}\end{array}$ & $\begin{array}{l}\text { Aplicar emolientes sobre a pele seca; cortar } \\
\text { as unhas de forma regular e correta; fazer a } \\
\text { proteção solar de forma regular }\end{array}$ & $\begin{array}{l}\text { Carta de Ottawa: Reconhecer a pessoa como o } \\
\text { principal recurso para a saúde } \\
\text { Declaração de Santafé de Bogotá: Impulsio- } \\
\text { nar a promoção da saúde } \\
\text { Declaração de Jacarta: Acumular conheci- } \\
\text { mentos sobre as melhores práticas }\end{array}$ \\
\hline $\begin{array}{l}\text { Ensaio clínico } \\
\text { randomizado }^{(10)}\end{array}$ & $\begin{array}{l}\text { Monitorizar a temperatura do pé no } \\
\text { domicílio }\end{array}$ & $\begin{array}{l}\text { Carta de Ottawa: Reconhecer a pessoa como } \\
\text { o principal recurso para a saúde; reorientar os } \\
\text { serviços de saúde para a promoção da saúde } \\
\text { Declaração de Jacarta: Acumular conhecimen- } \\
\text { tos sobre as melhores práticas. }\end{array}$ \\
\hline $\begin{array}{l}\text { Pesquisa de aborda- } \\
\text { gem qualitativa }{ }^{(11)}\end{array}$ & $\begin{array}{l}\text { Reduzir as horas de exposição ao sol; fazer } \\
\text { a proteção solar de forma regular }\end{array}$ & $\begin{array}{l}\text { Carta de Ottawa: Reconhecer a pessoa como o } \\
\text { principal recurso para a saúde } \\
\text { Declaração de Santafé de Bogotá: Impulsio- } \\
\text { nar a promoção da saúde } \\
\text { Declaração de Jacarta: Acumular conhecimen- } \\
\text { tos sobre as melhores práticas }\end{array}$ \\
\hline Estudo de $\mathrm{Caso}^{(5)}$ & $\begin{array}{l}\text { Não caminhar descalço; Usar sapatos bem ajus- } \\
\text { tados; verificar a parte de dentro dos sapatos } \\
\text { antes de usá-los; verificar os pés diariamente; } \\
\text { manter os pés limpos, secos ehidratados regu- } \\
\text { larmente; cortar as unhas de forma regular e } \\
\text { correta; evitar fontes diretas de calor }\end{array}$ & $\begin{array}{l}\text { Carta de Ottawa: Reconhecer a pessoa como o } \\
\text { principal recurso para a saúde } \\
\text { Declaração de Santafé de Bogotá: Impulsio- } \\
\text { nar a promoção da saúde } \\
\text { Declaração de Jacarta: Acumular conheci- } \\
\text { mentos sobre as melhores práticas }\end{array}$ \\
\hline Estudo de caso $^{(12)}$ & $\begin{array}{l}\text { Cortar as unhas de forma regular e correta; } \\
\text { retirar os móveis do percurso no ambiente } \\
\text { domiciliar e ter mais cuidado com objetos } \\
\text { pontiagudos e outros; realizar a ingestão de } \\
\text { líquidos para hidratar a pele }\end{array}$ & $\begin{array}{l}\text { Carta de Ottawa: Reconhecer a pessoa como o } \\
\text { principal recurso para a saúde } \\
\text { Declaração de Santafé de Bogotá: Impulsio- } \\
\text { nar a promoção da saúde } \\
\text { Declaração de Jacarta: Acumular conheci- } \\
\text { mentos sobre as melhores práticas }\end{array}$ \\
\hline $\begin{array}{l}\text { Revisão de litera- } \\
\text { tura }^{(13)}\end{array}$ & $\begin{array}{l}\text { Usar água morna ao invés de água quente } \\
\text { para se lavar; usar produtos que substituam } \\
\text { o sabão; aplicar emolientes sobre a pele seca; } \\
\text { usar uma toalha macia e suave acariciando } \\
\text { a pele seca; evitar a exposição a substâncias } \\
\text { irritantes; ter uma dieta saudável }\end{array}$ & $\begin{array}{l}\text { Carta de Ottawa: Reconhecer a pessoa como o } \\
\text { principal recurso para a saúde } \\
\text { Declaração de Santafé de Bogotá: Impulsio- } \\
\text { nar a promoção da saúde } \\
\text { Declaração de Jacarta: Acumular conheci- } \\
\text { mentos sobre as melhores práticas }\end{array}$ \\
\hline $\begin{array}{l}\text { Revisão de litera- } \\
\text { tura }^{(14)}\end{array}$ & $\begin{array}{l}\text { Avaliar diariamente a pele; ter uma dieta } \\
\text { saudável; aliviar a pressão da ferida e de } \\
\text { outras áreas de risco }\end{array}$ & $\begin{array}{l}\text { Carta de Ottawa: Reconhecer a pessoa como o } \\
\text { principal recurso para a saúde } \\
\text { Declaração de Santafé de Bogotá: Reorien- } \\
\text { tar os serviços de saúde para a promoção da } \\
\text { saúde; impulsionar a promoção da saúde. } \\
\text { Declaração de Jacarta: Acumular conheci- } \\
\text { mentos sobre as melhores práticas }\end{array}$ \\
\hline
\end{tabular}

Cogitare Enferm. 2012 Out/Dez; 17(4):767-74 
Os dados apresentados no quadro 2 evidenciam que a Carta de Otawa e a Declaração de Jacarta, apresentam recomendações, no contexto de educação em saúde, presentes em todos os estudos analisados. Referente à Carta de Otawa, destaca-se a recomendação: Reconhecer as pessoas como o principal recurso para a saúde; apoiá-las e capacitá-las para que se mantenham saudáveis a si próprias, às suas famílias e amigos. A Declaração de Jacarta por sua vez recomenda: a acumulação de conhecimentos sobre as melhores práticas.

\section{DISCUSSÃO}

A promoção da saúde é parte de uma concepção ampla do processo saúde-doença, propondo articulação de saberes entre profissionais da saúde e comunidade. Um destaque especial é dado à educação em saúde como fator causal de tal promoção, focando atividades dirigidas à transformação do indivíduo ou comunidade ${ }^{(8)}$.

Dessa forma, as Cartas de Promoção da Saúde apresentam estratégias para o desenvolvimento das atividades de educação em saúde. É nessa perspectiva que se buscou integrar as Cartas com as indicações de promoção da saúde elencadas pelos autores dos artigos que embasaram a presente revisão.

Dentro da educação em saúde, destaca-se a promoção da integridade da pele. Diante disso, a discussão ramifica-se em três vertentes: capacitação da população e dos serviços de saúde na perspectiva da promoção da saúde; o conceito de saúde como estratégia para modificação de fatores condicionantes; o acúmulo de conhecimento condicionando melhores práticas.

\section{Capacitação da população e dos serviços de saúde na perspectiva da promoção da saúde}

A Carta de Ottawa define promoção de saúde como conjunto de ações voltadas para prevenção de doenças e riscos individuais, considerando a influência de aspectos sociais, econômicos, políticos e culturais sobre as condições de vida e saúde ${ }^{(7)}$.

Assim, o primeiro compromisso para a promoção da saúde, dentro da perspectiva da educação em saúde, observada na Carta de Ottawa é: reconhecer as pessoas como o principal recurso para a saúde, apoiá-las e capacitá-las para que se mantenham saudáveis a si próprias, às suas famílias e amigos, através de financiamentos e/ou outras formas de apoio, e aceitar a comunidade como porta-voz essencial em matéria de saúde, condições de vida e bem-estar.
$\mathrm{Na}$ análise dos estudos, observou-se que tal recomendação esteve presente na totalidade dos artigos, fato que comprova sua relevância. Os autores de um dos estudos analisados ${ }^{(1)}$ relatam a importância da divulgação de cuidados com a pele para pacientes idosos pois, a partir da educação em saúde, é possível que haja maior promoção da integridade da pele. Traz como principais recomendações: aplicação de emolientes sobre a pele seca; corte das unhas de forma regular e correta; e uso regular de proteção solar.

A monitoração da temperatura do pé de pacientes diabéticos apresenta-se como uma nova evidência na tentativa de promover a integridade da pele nessa população. No estudo randomizado ${ }^{(10)}$ analisado, os autores buscam comprovar a importância da auto-monitoração da temperatura da pele pelo paciente, mediante medidas educativas fornecidas ao mesmo.

Com foco na elevada prevalência de câncer de pele em idosos, fator desencadeante para o rompimento da integridade da pele, outro estudo avaliado ${ }^{(11)}$ sugere como medidas educativas: orientação para a redução das horas de exposição ao sol e o uso regular de proteção solar.

Nessa perspectiva, a educação em saúde centrada no paciente portador de Diabetes mellitus tipo II é sugerida como fator relevante na manutenção da integridade da pele, tendo como medidas educativas: não caminhar descalço; usar sapatos bem ajustados; verificar dentro dos sapatos antes de usá-los; verificar seus pés diariamente procurando danos; manter os pés limpos, secos e hidratados regularmente; corte das unhas de forma regular e correta; e evitar fontes diretas de calor ${ }^{(5)}$.

Outros autores ${ }^{(12)}$ expõem a necessidade das ações educativas junto ao paciente, família e comunidade, sendo essencial no controle dessa enfermidade. Enfermidade essa cujas complicações estão estritamente ligadas ao déficit de conhecimento do auto-cuidado. As medidas educativas citadas foram: corte das unhas de forma regular e correta; retirar móveis do percurso no ambiente domiciliar; ter mais cuidado com objetos pontiagudos e outros; e realizar a ingestão de líquidos para hidratar a pele.

Em outro estudo ${ }^{(13)}$, medidas para promover a integridade da pele em idosos são propostas, a saber: usar água morna ao invés de água quente para se lavar; usar produtos que substituam o sabão; aplicar emolientes sobre a pele seca; usar uma toalha macia e suave acariciando a pele seca; evitar a exposição a substâncias irritantes, incluindo alguns perfumes, 
colônias e desodorantes; e ter uma dieta saudável.

Outros autores ${ }^{(14)}$ alertam que a úlcera por pressão, por levar a comorbidades, deve ser evitada a partir da educação em saúde do paciente, seus familiares ou cuidadores, enfatizando a importância de avaliação diária da pele, dieta saudável e alívio de pressão para a ferida e outras áreas de risco.

Dessa forma, evidencia-se a preocupação de pesquisadores em seguir a recomendação da Carta de Ottawa, reconhecendo as pessoas como principal recurso para a saúde e enfocando a importância de capacitá-las, dentro da perspectiva da educação em saúde, utilizando-se das medidas educativas com enfoque em uma saúde de qualidade. Proporciona-se, portanto, maior visibilidade ao potencial do envolvimento da comunidade na saúde, além do apoio à capacitação da mesma para o autocuidado.

Assim, o envolvimento comunitário na monitoração da temperatura do pé de pacientes diabéticos é destacado como essencial por um dos estudos anali$\operatorname{sados}^{(10)}$, bem como, o caráter evitável das úlceras por pressão através da reorientação da equipe de saúde e da orientação dos familiares dos pacientes ${ }^{(14)}$.

\section{O conceito de saúde como estratégia para modifi- cação de fatores condicionantes}

A Declaração de Santa Fé de Bogotá debateu a promoção da saúde na América Latina com foco central na interdependência da saúde e do desenvolvimento. A conferência enfatizou que o papel da promoção da saúde consiste em atuar como um agente de mudança que induza transformações radicais nas atitudes e condutas da população e seus dirigentes ${ }^{(7)}$.

Nessa perspectiva de relação mútua entre saúde e desenvolvimento, a Declaração de Santa Fé de Bogotá propõe as seguintes recomendações: impulsionar o conceito de saúde condicionada por fatores políticos, econômicos, sociais, culturais, ambientais, de conduta e biológicos, e a promoção da saúde como estratégia para modificar estes fatores condicionantes ${ }^{(7)}$. A influência destas recomendações foi constatada em medidas educativas de seis artigos que compõem a presente revisão.

A saúde é um conceito complexo e amplo em que diversos fatores estão conectados e, estando eles isoladamente ou interrelacionados, atuam modificando a saúde, seja ela de um paciente, uma comunidade ou de uma determinada área. A promoção da saúde surge em resposta a esse novo conceito, buscando estratégias neste setor para a melhoria da qualidade de vida da população ${ }^{(15)}$.
Sabe-se que para atingir determinado objetivo é necessário que os indivíduos adquiram controle e conhecimento acerca de seu estado de saúde, tornando-se como grande o desafio da promoção em saúde: o desenvolvimento de atividades dirigidas à transformação dos comportamentos dos indivíduos, focando nos seus estilos de vida ${ }^{(15)}$.

Os programas ou atividades de promoção da saúde tendem a concentrar-se em componentes educativos. Portanto, as medidas educativas propostas pelos autores dos estudos analisados, tais como: avaliação diária da pele, hidratação da pele, uso de filtro solar, dentre outras, tendem a modificar os estilos de vida dos pacientes e, consequentemente, os fatores condicionantes da saúde. Ao mesmo tempo, proporcionam melhoria na qualidade de vida destes, promovendo a integridade da pele e, desta forma, atendendo ao novo conceito amplo de saúde como sugerido na recomendação da Declaração de Santa Fé de Bogotá.

\section{O acúmulo de conhecimento condicionando melho- res práticas}

A Declaração de Jacarta trata da Quarta Conferência Internacional sobre Promoção da Saúde realizada na Indonésia em julho de 1997, e tem como enfoque a Promoção da Saúde no século XXI. Tal Declaração propõe novas ações, sendo estas: a cooperação internacional e a criação de novas parcerias, a defesa à participação do setor privado na promoção da saúde, a avaliação do aprendizado acumulado em promoção da saúde, os fatores determinantes da saúde e as estratégias necessárias para enfrentar os novos desafios ${ }^{(7)}$.

Na tentativa de acelerar o progresso para a promoção da saúde mundial, a Declaração de Jacarta define prioridades de ações neste sentido, dentre as quais destaca-se: acumulação de conhecimentos sobre as melhores práticas. Tal recomendação foi evidenciada na totalidade dos sete artigos utilizados como embasamento teórico científico da presente revisão.

As práticas de autocuidado proporcionam maior importância à saúde, uma vez que o paciente adquire autonomia e liberdade para com a sua saúde, garantindo-lhe melhorias na qualidade de vida. Nessa perspectiva, os autores dos artigos expõem medidas educativas que se caracterizam tanto pela fácil aplicabilidade quanto pela resolutividade na manutenção da integridade da pele. Assim sendo, os autores elegeram as melhores práticas de autocuidado, as quais devem ser transmitidas à população como medidas educativas de saúde. 
Em meio às medidas relacionadas à promoção da integridade da pele em pessoas idosas, e pensando também no contexto das Cartas de Promoção da Saúde, ressalta-se a preocupação dos referidos autores em fazer uso das recomendações preconizadas pelas Cartas. A partir da análise das Cartas foi possível destacar a preocupação com a educação em saúde, o que leva a compreender que tal intervenção é de grande valia para a promoção da mesma, ampliando-se o pensamento da necessidade cada vez maior de se promover saúde por meio da educação.

$\mathrm{Na}$ visão da enfermagem, essa necessidade se afirma no desenvolvimento de atividades educativas para aumentar o conhecimento dos pacientes e comunidade, procurando contribuir para a adesão do paciente ao tratamento. Destarte, é imprescindível que os profissionais da saúde, em especial os enfermeiros, direcionem sua prática para ações que levem à independência, à autonomia e à qualidade de vida dos idosos.

\section{CONCLUSÃO}

Na presente revisão buscou-se relacionar as medidas educativas para a promoção da integridade da pele em idosos com as Cartas de Promoção da Saúde, e a partir dessa relação contribuir para uma melhor prática dos profissionais da saúde, principalmente no que diz respeito à Enfermagem. As principais medidas educativas para a promoção da integridade pele em pessoas idosas encontradas nos artigos analisados foram: a inspeção diária da pele, os cuidados com calçados e com os pés, o uso regular de proteção solar e hidratante e as mudanças de decúbito para a redução da pressão nas proeminências ósseas.

Na conjuntura da promoção à saúde, observou-se que as medidas preconizadas pelos autores correlacionavam-se com as recomendações destacadas nas Cartas. Nesse âmbito, destaca-se a relevância dessa relação para a criação de uma cultura de saúde, com enfoque na população e no indivíduo como agentes executores imprescindíveis para o alcance da promoção da saúde. Nessa perspectiva, existe a necessidade de que os profissionais da saúde, principalmente o enfermeiro, realizem satisfatoriamente a inspeção da pele do idoso e o informe de todas as medidas de autocuidado, de maneira clara e detalhada.

Destarte, a promoção em saúde a partir das medidas educativas é de responsabilidade dos profissionais da área, principalmente devido o caráter evitável da maioria dos problemas causadores do rompimento da integridade da pele em indivíduos senis.

\section{REFERÊNCIAS}

1. Liao YH, Chen KH, Tseng MP, Sun CC. Pattern of skin diseases in a geriatric patient group in Taiwan: a 7-year survey from the outpatient clinic of a University medical Center. Dermatology. [Internet] 2001;203(4) [acesso em 05 nov 2010]. Disponível: http://content.karger.com/ ProdukteDB/produkte.asp?Aktion=ShowPDF\&Artike $1 \mathrm{Nr}=51778 \&$ Ausgabe $=227732 \&$ Produkt $\mathrm{Nr}=224164 \& \mathrm{f}$ ilename $=51778$.pdf.

2. Silva TL, Santos SSC, Pelzer MT, Barlem ELD, Arrieche TA. Conhecimento específico de enfermeiros de um hospital universitário acerca do cuidado ao idoso. Cogitare enferm. [Internet] 2009;14(1) [acesso em 02 nov 2010]. Disponível: http://ojs.c3sl.ufpr.br/ojs2/index. $\mathrm{php} /$ cogitare/article/view/14122/9493.

3. Bhutani T, Peng D, Shin T, Lee H. Common sources of skin care education: a cross-sectional study. Int $\mathrm{j}$ dermatol. [Internet] 2009;48(5) [acesso em 02 nov 2010]. Disponível: http://onlinelibrary.wiley.com/ doi/10.1111/j.1365-4632.2009.04040.x/pdf.

4. Rezende DM, Bachion MM, Araújo LAO. Integridade da pele prejudicada em idosos: estudo de ocorrência numa comunidade atendida pelo programa saúde da família. Acta Paul. Enferm. [Internet] 2006;19(2) [acesso em 02 nov 2010]. Disponível: http://www.scielo. br/pdf/ape/v19n2/a08v19n2.pdf.

5. Fletcher J. Full nursing assessment of patients at risk of diabetic foot ulcers. Br $\mathrm{j}$ nurs. [Internet] 2006;15(15) [acesso em 05 nov 2010]. Disponível: http://www.internurse.com/cgibin/go.pl/library/article. cgi?uid=21696; article=BJN_15_15\%20Suppl_S18_ S21;format $=$ pdf.

6. Resnick B. Promoting health in older adults: a fouryear analysis. J Am Acad Nurse Pract. [Internet] 2001;13(1) [acesso em 02 dez 2010]. Disponível: http:// onlinelibrary.wiley.com/doi/10.1111/j.1745-7599.2001. tb00212.x/pdf.

7. Ministério da Saúde (Br). Secretaria de Políticas de Saúde. Projeto Promoção da Saúde. Cartas de Promoção da Saúde. Série: B. Textos Básicos em Saúde. Brasília: Ministério da Saúde; 2002.

8. Buss PM. Promoção da saúde e qualidade de vida. Ciênc. saúde colet. [Internet] 2000;5(1) [acesso em 02 nov 2010]. Disponível: http://www.scielo.br/pdf/csc/ v5n1/7087.pdf. 
9. Leopardi MT. Metodologia da pesquisa em saúde. $2^{\mathrm{a}} \mathrm{ed}$. Florianópolis: UFCS/ Pós-Graduação em Enfermagem; 2002.

10. Lavery LA, Higgins KR, Lanctot DR, Constantinides GP, Zamorano RG, Armstrong DG, et al. Home monitoring of foot skin temperatures to prevent ulceration. Diabetes care. [Internet] 2004;27(11) [acesso em 05 nov 2010]. Disponível: http://care. diabetesjournals.org/content/27/11/2642.full.pdf.

11. Carmel S, Shani E, Rosenberg L. The role of age and an expanded health belief model in predicting skin cancer protective behavior. Health Educ Res. [Internet] 1994;9(4) [acesso em 05 nov 2010]. Disponível: http:// www.ncbi.nlm.nih.gov/pubmed/10150459.

12. Faeda A, Leon CGRMP. Assistência de enfermagem a um paciente portador de Diabetes Mellitus. Rev. Bras. Enferm. [Internet] 2006;59(6) [acesso em 05 nov 2010]. Disponível: http://www.scielo.br/pdf/reben/v59n6/a19. pdf

13. Cowdell F. Promoting skin health in older people. Nurs older people. [Internet] 2010;2(10) [acesso em 05 nov 2010]. Disponível: http://www.ncbi.nlm.nih.gov/ pubmed/21197892

14. Brem H, Lyder C. Protocol for the successful treatment of pressure ulcers. Am J Surg. [Internet] 2004;188(1A Suppl) [acesso em 05 nov 2010]. Disponível: http://www. ncbi.nlm.nih.gov/pubmed/15223496.

15. Czeresnia D, Freitas CM. Promoção da saúde: conceitos, reflexões, tendências. Rio de Janeiro: Fiocruz; 2003. 